\title{
Design, synthesis and biological activity of hydroxybenzoic acid ester conjugates of phenazine-1-carboxylic acid
}

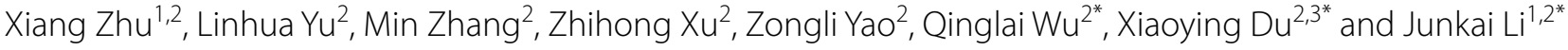

\begin{abstract}
We prepared 16 novel hydroxybenzoic acid ester conjugates of phenazine-1-carboxylic acid (PCA) and investigated their biological activity. Most of the synthesized conjugates displayed some level of fungicidal activities in vitro against five phytopathogenic fungi. Nine conjugates $\mathbf{5 b}, \mathbf{5 c}, \mathbf{5 d}, \mathbf{5 e}, \mathbf{5 h}, \mathbf{5 i}, \mathbf{5 m}, \mathbf{5 n}$ and $\mathbf{5 0}\left(\mathrm{EC}_{50}\right.$ between $3.2 \mathrm{\mu g} / \mathrm{mL}$ and $14.1 \mu \mathrm{g} / \mathrm{mL}$ ) were more active than PCA (EC $18.6 \mu \mathrm{g} / \mathrm{mL}$ ) against Rhizoctonia solani. Especially conjugate $\mathbf{5 c}$ showed the higher fungicidal activity against Rhizoctonia solani which is 6.5 -fold than PCA. And the results of the bioassay indicated that the fungicidal activity of conjugates was associated with their LogP, and the optimal LogP values of the more potent fungicidal activities within these conjugates ranged from 4.42 to 5.08 . The systemic acquired resistance induced by PCA-SA ester conjugate $\mathbf{5} \mathbf{c}$ against rice sheath blight disease in rice seedlings was evaluated. The results revealed that PCA-SA ester conjugate $\mathbf{5} \mathbf{c}$ retained the resistance induction activity of SA against rice sheath blight.
\end{abstract}

Keywords: Phenazine-1-carboxylic acid, Synthesis, Biological activity, Salicylic acid

\section{Background}

Phenazine-1-carboxylic acid (PCA) (1, Fig. 1) is a secondary metabolite isolated from Pseudomonas, Streptomycetes, and a few other bacterial genera from soil or marine habitats [1-5]. The biological properties of PCA includes antimicrobial [6-9] antiviral [7], antitumorigenic [8-12] antitubercular and antileukemic activities $[13,14]$. In China, PCA has been registered as a biofungicide against rice sheath blight caused by Rhizoctonia solani, and it is noted for its high efficacy, low toxicity, environmental friendliness and enhancement of crop production [15-18]. PCA is also an important precursor for the biosynthesis of ester derivatives $[1,19]$, some of which show higher fungicidal activity against several phytopathogenic fungi. For instance, compound 6 (Fig. 1) isolated from Pseudomonas, was a more effective derivative against Alternaria alternata and R. solani than PCA

\footnotetext{
*Correspondence: wql106@163.com; Qinger539@163.com; junkaili@sina. com

${ }^{2}$ School of Agriculture, Yangtze University, Jingmi Road 88, Jingzhou 434025, China

Full list of author information is available at the end of the article
}

[5]. As reported, some synthetic phenazine-1-carboxylate derivatives prepared by chemical modification of the carboxyl group with various alkyl alcohols exhibit strong fungicidal activity against Pyricularia oryzae, and in particular the inhibition of derivative 7 was $100 \%$ complete at $8.3 \mu \mathrm{g} / \mathrm{mL}$ [20]. Recently, a series of novel aminophenazine-1-carboxylate derivatives were synthesized and evaluated against five fungi [21], and the results of bioassay showed that compounds $\mathbf{8}$ and $\mathbf{9}$ (Fig. 1) could exhibited strong activity against $P$. piricola with $\mathrm{EC}_{50}$ values of $3.00 \mu \mathrm{g} / \mathrm{mL}$ and $4.44 \mu \mathrm{g} / \mathrm{mL}$ respectively, which were both lower than that of PCA.

Salicylic acid (SA) (Fig. 2), also known as o-hydroxybenzoic acid which is one of the three isomers of hydroxybenzoic acid, is an important plant growth regulator playing a role in the hypersensitive reaction (HR) and acts as an endogenous signal responsible for inducing systemic acquired resistance in plants $[22,23]$. The plants treated with salicylic acid or its derivatives may be able to resist infection by various plant pathogens [24-26]. Hydroxybenzoate esters, which are widely used in medicine, foods and cosmetics, have been reported to have various biological activities, such as antimicrobial 
<smiles>[R]C([R])c1cccc2nc3ccccc3nc12</smiles>

$1 \mathrm{R}=\mathrm{OH}$ $6 \mathrm{R}=\mathrm{OMe}$<smiles>O=C(OC1CCCCC1)c1cccc2nc3ccccc3nc12</smiles>

7

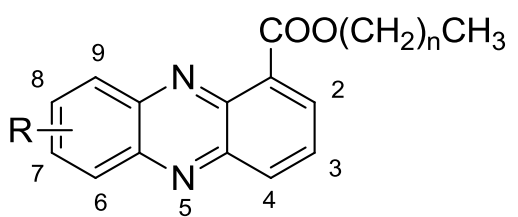

$8 \mathrm{R}=6-\mathrm{NH}_{2}, \mathrm{n}=0$ $9 \mathrm{R}=6-\mathrm{NH}_{2}, \mathrm{n}=1$

Fig. 1 The structures of PCA and its derivatives<smiles>[R10]C(=O)c1ccccc1OC(=O)c1cccc2nc3ccccc3nc12</smiles>

5a $\mathrm{R}^{1}=\mathrm{CH}_{3}$

5b $\mathrm{R}^{1}=\mathrm{CH}_{2} \mathrm{CH}_{3}$

5c $\mathrm{R}^{1}=\left(\mathrm{CH}_{2}\right)_{2} \mathrm{CH}_{3}$

5d $\mathrm{R}^{1}=\mathrm{CH}\left(\mathrm{CH}_{3}\right)_{2}$

5e $\mathrm{R}^{1}=\left(\mathrm{CH}_{2}\right)_{3} \mathrm{CH}_{3}$<smiles>[R]OC(=O)c1cccc(OC(=O)c2cccc3nc4ccccc4nc23)c1</smiles><smiles>[R]OC(=O)c1ccc(OC(=O)c2cccc3nc4ccccc4nc23)cc1</smiles>

5f $\mathrm{R}^{2}=\mathrm{CH}_{3}$

5k $\mathrm{R}^{3}=\mathrm{CH}_{3}$

5g R ${ }^{2}=\mathrm{CH}_{2} \mathrm{CH}_{3}$

5I $\mathrm{R}^{3}=\mathrm{CH}_{2} \mathrm{CH}_{3}$

5h $\mathrm{R}^{2}=\left(\mathrm{CH}_{2}\right)_{2} \mathrm{CH}_{3}$

5i $\mathrm{R}^{2}=\mathrm{CH}\left(\mathrm{CH}_{3}\right)_{2}$

$5 \mathrm{~m} \mathrm{R}^{3}=\left(\mathrm{CH}_{2}\right)_{2} \mathrm{CH}_{3}$

5n $\mathrm{R}^{3}=\mathrm{CH}\left(\mathrm{CH}_{3}\right)_{2}$

5j $\mathrm{R}^{2}=\left(\mathrm{CH}_{2}\right)_{3} \mathrm{CH}_{3}$ 5o $\mathrm{R}^{3}=\left(\mathrm{CH}_{2}\right)_{3} \mathrm{CH}_{3}$

$5 p \mathrm{R}^{3}=\left(\mathrm{CH}_{2}\right)_{7} \mathrm{CH}_{3}$

Fig. 2 The structures of PCA-salicylic acid ester conjugates (5a-5e), PCA-3-hydroxybenzoic acid ester conjugates $(\mathbf{5} \mathbf{f}-\mathbf{5} \mathbf{j})$ and PCA-p-hydroxybenzoic acid ester conjugates $(\mathbf{5 k}-\mathbf{5} \mathbf{p})$

[27-29] antiviral [30, 31], anti-inflammatory and nematicidal activities [32], among others. Accordingly, hydroxybenzoate esters with multiple bioactive chemical structures, have drawn wide attention in the biological and pharmacological fields.

In this research, considering the potential biological activity of phenazine-1-carboxylic derivatives and that there have been few published studies on the biological activity of phenazine-1-carboxylic phenolic esters, we designed and synthesized 16 novel phenolic ester derivatives of phenazine-1-carboxylic acid (Fig. 2) by a simple esterification reaction of PCA and three types of hydroxybenzoic acids. To enhance the lipophilic properties of the these conjugates, hydroxybenzoic acids were derivatized to its ester with the corresponding $\mathrm{CH}_{3}\left(\mathrm{CH}_{2}\right) \mathrm{nOH}$. The synthetic route of conjugates $\mathbf{5 a}-\mathbf{5 p}$ is described in Fig. 3. All these conjugates were evaluated for their fungicidal activity against five phytopathogenic fungi in vitro. Furthermore, the systemic acquired resistance of the most active PCA-SA ester conjugate 5c against rice sheath blight disease was also investigated in rice plants.

\section{Results and discussion Chemistry}

As shown in Fig. 3, three types of hydroxybenzoate esters (4) were first synthesized by a simple esterification reaction with 2-hydroxybenzoic acid, 3-hydroxybenzoic acid or 4-hydroxybenzoic acid as the starting materials. Then treatment of PCA with oxalyl chloride at the reflux temperature in $\mathrm{CH}_{2} \mathrm{Cl}_{2}$ solution afforded intermediate 2 after the evaporation of $\mathrm{CH}_{2} \mathrm{Cl}_{2}$. The target compound 5 a was synthesized by adding intermediate 2 to compound $4 \mathbf{a}$ in $\mathrm{CH}_{2} \mathrm{Cl}_{2}$ solution, stirred at room temperature for $2 \mathrm{~h}$. PCA-salicylic acid ester conjugates $(\mathbf{5 a} \mathbf{a}-\mathbf{5 e})$, PCA-3-hydroxybenzoic acid ester conjugates $(\mathbf{5} \mathbf{f}-\mathbf{5 j})$ and 


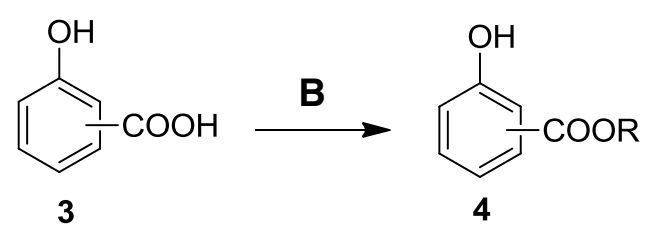<smiles>[R]Oc1ccc(OC(=O)c2cccc3nc4ccccc4nc23)cc1</smiles>

Fig. 3 Synthetic route of target compounds. Reagents and conditions: a oxalyl chloride, $\mathrm{CH}_{2} \mathrm{Cl}_{2}, \mathrm{DMF}$, reflux, 8 h; b alcohol, reflux, overnight; $\mathbf{c}$ hydroxybenzoic acid ester, $\mathrm{CH}_{2} \mathrm{Cl}_{2}$, room temperature to reflux, $2 \mathrm{~h}$

PCA-p-hydroxybenzoic acid ester conjugates (5k-5p) were synthesized by this method.

The structures of all conjugates were characterized by ${ }^{1} \mathrm{H}$ NMR and high resolution mass spectroscopy (HRMS) analyses, and the representative conjugate $\mathbf{5 d}$ was confirmed by the X-ray crystallographic analysis. The molecular structure of $\mathbf{5 d}$ is shown in Fig. 4. The crystal data for 5d: triclinic, space group $\mathrm{P} 2 / 1 / \mathrm{c}, \mathrm{a}=18.130$ (3) $\AA$, $\mathrm{b}=12.258$ (2) $\AA, \mathrm{c}=8.6490$ (14) $\AA, \mathrm{a}=90^{\circ}, \mathrm{b}=96.224$ $(3)^{\circ}, \mathrm{g}=90^{\circ}, \mathrm{V}=1910.7$ (6) $\AA 3, \mathrm{Z}=4, \mathrm{~T}=297$ (2) $\mathrm{K}, \mu$ $(\mathrm{Mo})=0.093 \mathrm{~mm}^{-1}, \mathrm{D}_{\text {calcd. }}=1.343 \mathrm{Mg} / \mathrm{m}^{3}, 14,129$ reflections measured $\left(1.130 \leq 2 \Theta \leq 26.000^{\circ}\right), 3755$ unique $(R$ (int) $=0.0316)$ which were used in all calculations. The final $R_{1}$ was 0.0408 (I $>2$ sigma (I)) and $w R_{2}$ was 0.1162 . Crystallographic data have been deposited with the Cambridge Crystallographic Data Centre, and the deposition number was CCDC 1563918 (Additional file 1).

\section{Fungicidal activities}

All novel conjugates $(\mathbf{5 a}-\mathbf{5 p})$ were primarily screened in vitro against five phytopathogenic fungi, $R$. solani, $A$. solani, Fusarium oxysporum, Fusaium graminearum and P. oryzae, with PCA as a control. The results of the preliminary bioassay are shown in Table 1 . We found that

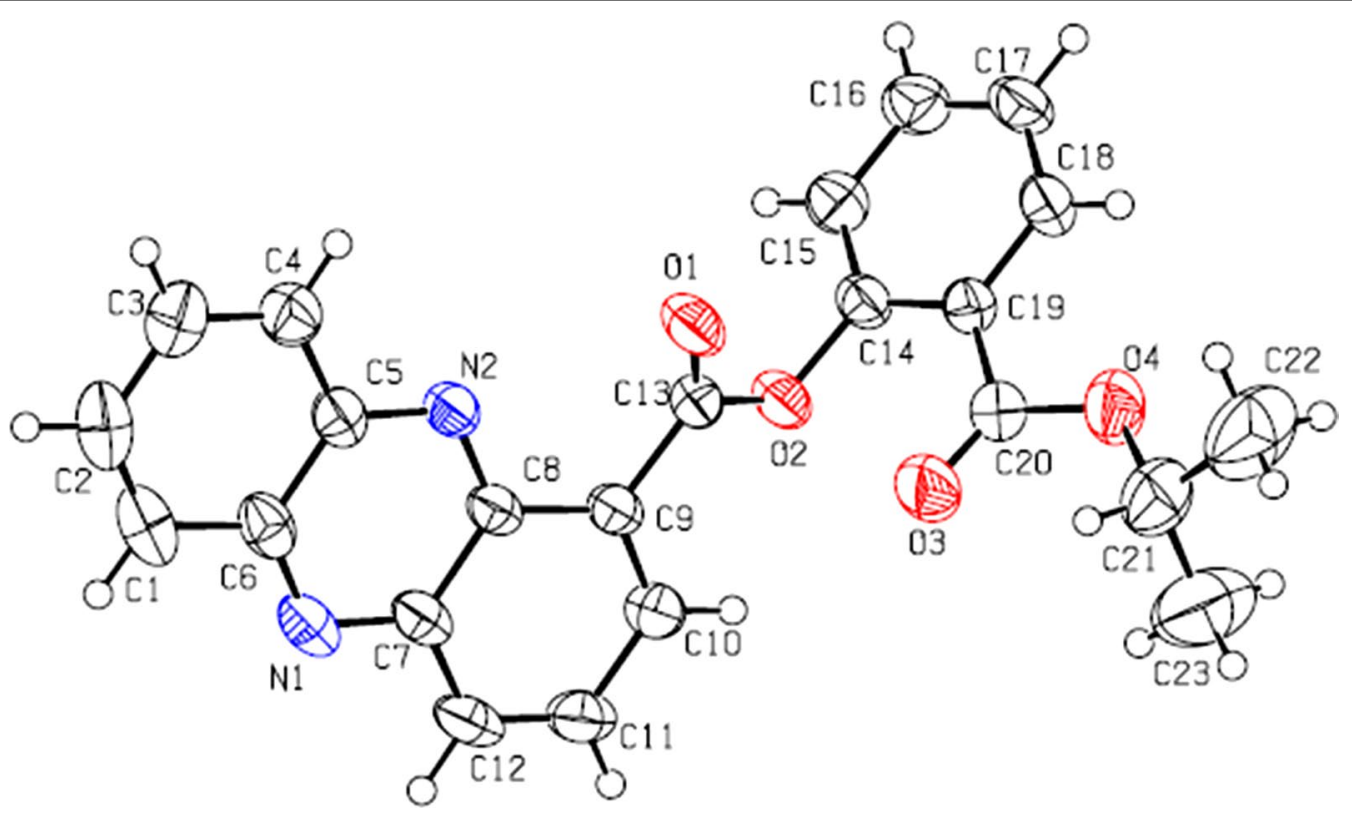

Fig. 4 The crystal structure of conjugate $\mathbf{5 d}$ 
Table 1 Fungicidal activity of compounds $5 \mathrm{a}-5 \mathrm{p}$ against five plant fungi in vitro at $50 \mu \mathrm{g} / \mathrm{mL}$ (inhibition rate/\%)

\begin{tabular}{|c|c|c|c|c|c|}
\hline Compd. & R. solani & A. solani & F. oxysporum & F. graminearum & P. oryzae \\
\hline $5 a$ & $66.2 \pm 1.5$ & $11.7 \pm 0.5$ & $13.1 \pm 0.6$ & $7.3 \pm 0.9$ & $34.5 \pm 0.9$ \\
\hline $5 b$ & $91.6 \pm 0.8$ & $30.3 \pm 1.6$ & $15.7 \pm 1.3$ & $15.9 \pm 2.6$ & $32.8 \pm 0.0$ \\
\hline $5 c$ & $100.0 \pm 0.0$ & $13.0 \pm 2.3$ & $12.4 \pm 0.8$ & $6.5 \pm 0.2$ & $37.0 \pm 2.7$ \\
\hline $5 d$ & $93.5 \pm 0.6$ & $12.4 \pm 0.9$ & $31.4 \pm 2.9$ & $12.3 \pm 1.3$ & $27.0 \pm 1.2$ \\
\hline $5 e$ & $93.1 \pm 0.9$ & $15.1 \pm 0.6$ & $9.8 \pm 0.3$ & $10.9 \pm 0.6$ & $27.0 \pm 3.9$ \\
\hline $5 f$ & $37.3 \pm 1.2$ & $45.5 \pm 0.3$ & $35.3 \pm 3.4$ & $13.0 \pm 0.9$ & $72.3 \pm 0.0$ \\
\hline $5 \mathrm{~g}$ & $41.2 \pm 0.8$ & $21.3 \pm 1.3$ & $16.3 \pm 0.9$ & $11.6 \pm 2.7$ & $49.6 \pm 2.6$ \\
\hline $5 \mathrm{~h}$ & $93.2 \pm 0.3$ & $15.1 \pm 0.5$ & $9.8 \pm 0.5$ & $10.9 \pm 3.4$ & $27.0 \pm 0.9$ \\
\hline $5 i$ & $100.0 \pm 0.0$ & $28.9 \pm 1.8$ & $18.3 \pm 2.7$ & $10.1 \pm 0.5$ & $45.4 \pm 1.2$ \\
\hline $5 \mathbf{j}$ & $45.1 \pm 1.0$ & $17.2 \pm 2.5$ & $13.1 \pm 0.5$ & $11.6 \pm 1.9$ & $39.6 \pm 1.5$ \\
\hline $5 k$ & $33.9 \pm 0.9$ & $18.6 \pm 0.3$ & $11.1 \pm 0.6$ & $8.7 \pm 3.5$ & $39.6 \pm 3.7$ \\
\hline 51 & $42.4 \pm 1.2$ & $19.3 \pm 0.9$ & $13.7 \pm 1.1$ & $13.0 \pm 4.4$ & $45.4 \pm 0.9$ \\
\hline $5 \mathrm{~m}$ & $100.0 \pm 0.0$ & $24.1 \pm 1.5$ & $15.7 \pm 1.6$ & $10.9 \pm 0.8$ & $39.6 \pm 0.2$ \\
\hline $5 n$ & $98.3 \pm 0.2$ & $26.2 \pm 0.9$ & $13.7 \pm 1.5$ & $8.7 \pm 4.6$ & $41.2 \pm 0.9$ \\
\hline 50 & $93.0 \pm 0.2$ & $15.1 \pm 0.5$ & $9.8 \pm 2.9$ & $10.9 \pm 3.3$ & $27.0 \pm 0.8$ \\
\hline $5 p$ & $44.5 \pm 1.2$ & $18.6 \pm 0.9$ & $13.7 \pm 0.9$ & $0 \pm 0.0$ & $34.5 \pm 4.9$ \\
\hline PCA & $86.2 \pm 0.9$ & $85.2 \pm 1.2$ & $83.5 \pm 1.9$ & $86.1 \pm 1.9$ & $92.0 \pm 2.7$ \\
\hline
\end{tabular}

Each treatment had three replicates (Mean $\pm S D$ ). The phenazine-1 carboxylic acid (PCA) was used as the positive control

most of conjugates $(\mathbf{5 a}-\mathbf{5 p})$ showed low activities against A. solani, F. oxysporum, F. graminearum and P. oryzae Cavara at a concentration of $50 \mu \mathrm{g} / \mathrm{mL}$, while most conjugates $(\mathbf{5 a}-\mathbf{5 p})$ exhibited high activity against $R$. solani at that rate. The inhibitory activity of $5 \mathbf{c}, 5 \mathbf{e}, 5 \mathbf{i}$ and $5 \mathrm{~m}$ was $100 \%$, higher than PCA at $86.2 \%$. To more closely examine preliminary structure-activity relationships (SARs), the conjugates $(\mathbf{5} \mathbf{a}-\mathbf{5 p})$ were selected for assessment of $\mathrm{EC}_{50}$ values against Rhizoctonia solani.

The $\mathrm{EC}_{50}$ values against Rhizoctonia solani for all conjugates are presented in Table 2 . The results showed that nine conjugates $(\mathbf{5 b}, \mathbf{5 c}, \mathbf{5 d}, \mathbf{5 e}, \mathbf{5 h}, \mathbf{5 i}, \mathbf{5 m}, \mathbf{5 n}$ and $\mathbf{5 0}$ ) with $\mathrm{EC}_{50}$ values between 3.2 and $14.1 \mu \mathrm{g} / \mathrm{mL}$ exhibited more potent fungicidal activity against Rhizoctonia solani than PCA $\left(\mathrm{EC}_{50}=18.6 \mu \mathrm{g} / \mathrm{mL}\right)$. In particular, conjugate 5c with highest fungicidal activity was 6.5-fold more active than PCA.

The recent study on fungicidal mechanism of PCA indicate that, PCA will promote cell produces poisonous hydroxyl radical and disrupt the normal homeostasis of redox in cells after entering cells through cell walls and cell membranes $[19,33]$. It means that a PCA analog with suitable polarity and hydrophobicity can pass through the cell membranes of pathogenic bacteria and fungi more easily and exhibit higher biological activity. As can be seen from Table 2, the fungicidal activities of conjugates were associated with their LogP values. Accordingly, we constructed a mathematical model that described the LogP of conjugates that might be expected to produce high or low levels of fungicidal activity. From Fig. 5,
Table 2 EC $_{50}$ values against Rhizoctonia solani and octanolwater partition coefficient of conjugates $5 a-5 p$

\begin{tabular}{llll}
\hline Compd. & EC $_{\mathbf{5 0}}(\boldsymbol{\mu g} / \mathbf{m L})$ & Toxicity index & LogP' \\
\hline $\mathbf{5 a}$ & 48.3 & 0.43 & 3.84 \\
$\mathbf{5 b}$ & 14.1 & 1.48 & 4.42 \\
$\mathbf{5 c}$ & 3.2 & 6.50 & 4.72 \\
$\mathbf{5 d}$ & 9.5 & 2.19 & 4.75 \\
$\mathbf{5 e}$ & 12.8 & 1.63 & 5.08 \\
$\mathbf{5 f}$ & 96.3 & 0.22 & 3.91 \\
$\mathbf{5 g}$ & 68.6 & 0.30 & 4.34 \\
$\mathbf{5 h}$ & 9.5 & 2.19 & 4.81 \\
$\mathbf{5 i}$ & 4.9 & 4.24 & 4.77 \\
$\mathbf{5 j}$ & 56.9 & 0.37 & 5.02 \\
$\mathbf{5 k}$ & 138.4 & 0.15 & 3.92 \\
$\mathbf{5 l}$ & 70.5 & 0.30 & 4.37 \\
$\mathbf{5} \mathbf{m}$ & 4.5 & 4.62 & 4.86 \\
$\mathbf{5 n}$ & 5.6 & 3.71 & 4.75 \\
$\mathbf{5 0}$ & 11.8 & 1.76 & 5.05 \\
$\mathbf{5 p}$ & 70 & 0.30 & 6.46 \\
$\mathbf{P C A}$ & 18.6 & 1.00 & 1.59 \\
\hline
\end{tabular}

1 Partition coefficient "LogP" values were calculated using the ALOGPS 2.1 program

with increasing $\log \mathrm{P}$ values, the fungicidal activities of conjugates were also observed to increase. For instance, the LogP values of PCA-salicylic acid ester conjugates were ranked as follows: $\mathbf{5 a}<\mathbf{5 b}<\mathbf{5} \mathbf{c}$, and the fungicidal 


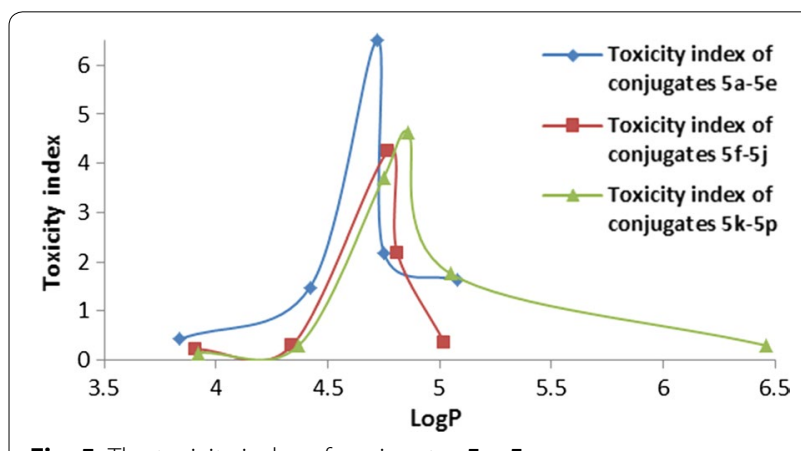

Fig. 5 The toxicity index of conjugates $\mathbf{5 a}-\mathbf{5 p}$

activity of conjugates also showed the same ranking. However, the conjugates that exceeded a certain level of LogP values (>4.72) had decreased fungicidal activity. For instance, the LogP values of PCA-salicylic acid ester conjugates were ranked $\mathbf{5 c}<\mathbf{5 d}<\mathbf{5 e}$, but the fungicidal activity of conjugates were ranked $\mathbf{5 c}>\mathbf{5 d}>\mathbf{5 e}$. The same trends also applied to the PCA-3-hydroxybenzoic acid ester conjugates $(\mathbf{5} \mathbf{f}-\mathbf{5 j})$ and the PCA- $p$-hydroxybenzoic acid ester conjugates (5k-5p). Through the above analysis, we found that the $\operatorname{LogP}$ values of the more potent fungicidal activity within these three types of conjugates ranged from 4.42 to 5.08 . Furthermore, conjugates where phenolic ester groups were substituted at different positions did not greatly affect their fungicidal activity.

\section{Systemic acquired resistance}

To evaluate the level of systemic acquired resistance induced by PCA-SA ester conjugates, the disease reduction of the most active PCA-SA ester conjugate 5c was investigated against rice sheath blight disease on rice seedlings following Makandar and others [34, 35]. The results of the study indicated that inoculation with conidia of Rhizoctonia solani onto rice plants treated with
SA and conjugate $\mathbf{5 c}$ resulted in fewer lesions per leaf sheath as well as reduced blighted leaf area as compared to control plants only receiving distilled water treatment (Fig. 6). Spray treatment with SA and PCA-SA ester conjugate $\mathbf{5 c}$ induced resistance to sheath blight disease in rice plants, significantly reducing rice sheath blight disease in rice plants. Compared with the treatments of PCA and water control, combined SA and conjugate 5c treatments had higher induction effects, at $31.0 \%$ and $57.0 \%$ respectively (Table 3 ).

At present, there is extensive research on possible structure-activity relationship of SA and its derivatives for induction of systemic acquired resistance. Safari assessed the potential of some chemical inducers of systemic acquired resistance (SAR) to reduce Alternaria leaf spot disease on tomato in glasshouse trials [26]. The results indicated that, among the salicylate derivatives, the biochemical activators containing electron donating groups are more suitable for inducing disease resistance in tomato crop. Also the structure relationship of 47 mono-substituted and multi-substituted salicylate derivatives with respect to their effects on disease resistance to tobacco mosaic virus and pathogenesis-related protein (PR1) accumulation were evaluated [25]. In this study, using this characteristic of SA, we demonstrated

\section{Table 3 Induced resistance of rice to rice sheath blight by different inducers treatment}

\begin{tabular}{lll}
\hline Inducers treatment & Disease (\%) & $\begin{array}{l}\text { Induced } \\
\text { effect } \\
\text { (\%) }\end{array}$ \\
\hline $\mathrm{A}(\mathrm{PCA})$ & 47.9 & 12.59 \\
$\mathrm{~B}(\mathrm{SA})$ & 37.3 & 31.97 \\
$\mathrm{C}$ (conjugate 5c) & 23.6 & 56.98 \\
D (water) & 54.8 & - \\
\hline
\end{tabular}

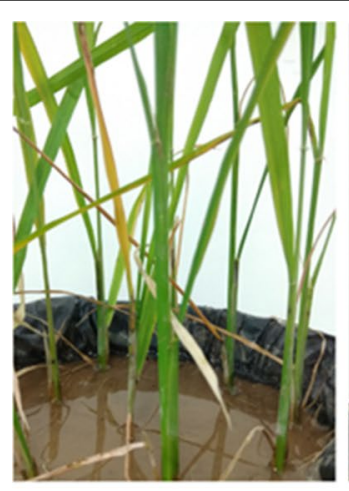

PCA

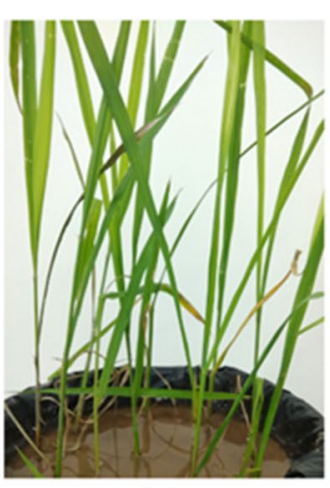

SA

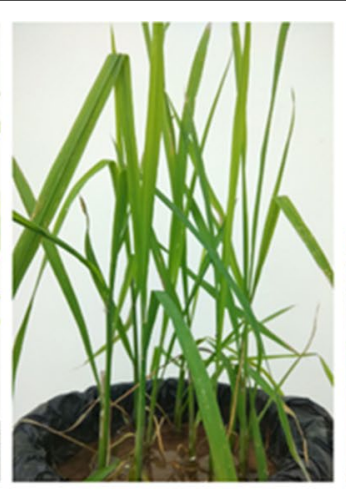

5c

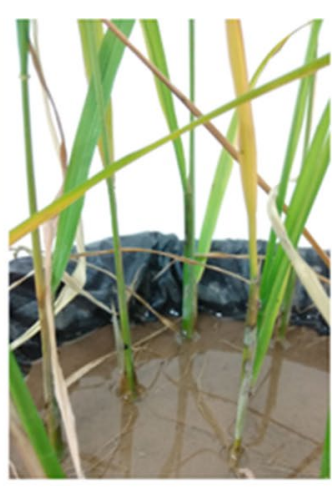

Water

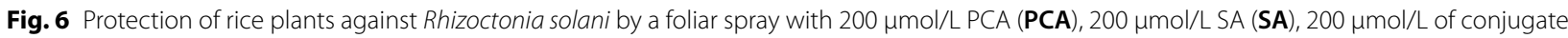
$\mathbf{5 c}(\mathbf{5 c})$ and distilled water (water) 14 days after inoculation with Rhizoctonia solani conidial suspension $\left(10^{5} \mathrm{spore} / \mathrm{mL}\right)$ 
that PCA-SA ester conjugate $5 \mathrm{c}$ retained the resistance induction activity of SA against rice sheath blight and had higher induced resistance than SA. However, the relationship between the structures of PCA-SA ester conjugates described here and their induced activities needs further investigation, as well as the mode of action.

\section{Experimental}

\section{Chemicals and instruments}

All chemicals and solvents were obtained from commercial suppliers and were used without further purification. The melting points were determined on a WRR melting point apparatus (Shanghai Jingke Industrial Co. Ltd., PR China) and were uncorrected. Thin-layer chromatography (TLC) was performed on silica gel 60 F254 (Qingdao Marine Chemical Ltd., P. R. China). Column chromatography (CC) purification was performed over silica gel (200-300 mesh, Qingdao Marine Chemical Ltd.). ${ }^{1} \mathrm{H}$ NMR spectrum were recorded in $\mathrm{CDCl}_{3}$ solution on a Bruker $600 \mathrm{MHz}$ spectrometer (Bruker Co., Switzerland), using tetramethylsilane (TMS) as an internal standard, and chemical shift values $(\delta)$ were given in parts per million (ppm). The following abbreviations were used to designate chemical shift multiplicities: $s=$ singlet, $\mathrm{d}=$ doublet, $\mathrm{t}=$ triplet, $\mathrm{q}=$ quartet, $\mathrm{m}=$ multiple. $M S$ data were obtained using a APEX IV Fourier-transform mass spectrometry (Bruker).

\section{Synthesis of hydroxybenzoic acid esters}

The compound 2-hydroxybenzoic acid $(15 \mathrm{mmol})$ and its corresponding alcohol $(30 \mathrm{~mL})$ were added into a $50 \mathrm{~mL}$ round-bottom flask, and cooled at $0{ }^{\circ} \mathrm{C}$. An aliquot of $2 \mathrm{~mL}$ of $98 \% \mathrm{H}_{2} \mathrm{SO}_{4}$ was slowly added. The reaction was stirred at reflux temperature for $12 \mathrm{~h}$ and monitored by thin-layer chromatography (TLC) until the 2-hydroxybenzoic acid was completely consumed. The mixture was evaporated under vacuum, neutralized with water and $5 \% \mathrm{NaHCO}_{3}$ aqueous solution, extracted by ether 3 times, dried over $\mathrm{Na}_{2} \mathrm{SO}_{4}$, concentrated in vacuum, and used in next step without purification. The compounds 3-hydroxybenzoic acid esters and $p$-hydroxybenzoic acid esters were also synthesized by this method.

\section{Synthesis of phenazine-1-carbonyl chloride}

Phenazine-1-carboxylic acid (10 mmol) and $N, N$-dimethylformamide $(0.1 \mathrm{mmol})$ were added in $30 \mathrm{~mL}$ of dry $\mathrm{CH}_{2} \mathrm{Cl}_{2}$, and cooled at $0{ }^{\circ} \mathrm{C}$. A solution of $15 \mathrm{mmol}$ of oxalyl chloride in $20 \mathrm{~mL}$ of dry $\mathrm{CH}_{2} \mathrm{Cl}_{2}$ was then slowly added. The reaction was stirred at reflux temperature for $12 \mathrm{~h}$, then cooled to room temperature and evaporated under vacuum. The residue was dissolved in $10 \mathrm{~mL}$ of dry $\mathrm{CH}_{2} \mathrm{Cl}_{2}$ and used in next step without purification.

\section{General procedure for hydroxybenzoic acid ester conjugates of phenazine-1-carboxylic acid 5a-5p} Phenazine-1-carbonyl chloride $(10 \mathrm{mmol})$ dissolved in $10 \mathrm{~mL}$ of dry $\mathrm{CH}_{2} \mathrm{Cl}_{2}$ was added dropwise to a solution of compound 2-hydroxybenzoic acid methyl ester $(10 \mathrm{mmol})$, and triethylamine $(12 \mathrm{mmol})$ as the attaching acid agent in $\mathrm{CH}_{2} \mathrm{Cl}_{2}$, The mixture was stirred at room temperature for $4 \mathrm{~h}$ until the reaction was complete (indicated by TLC), then quenched with water and $5 \% \mathrm{Na}_{2} \mathrm{CO}_{3}$ aqueous solution, dried over $\mathrm{Na}_{2} \mathrm{SO}_{4}$, filtered and concentrated in vacuum. The obtained crude extract was purified by recrystallizing from the solution of EtOAc-DCM (1:1) to give pure conjugate $\mathbf{5 a}$. Conjugates $\mathbf{5 b} \mathbf{- 5 p}$ were also synthesized by this method.

2-(Methoxycarbonyl)phenyl phenazine-1-carboxylate (5a) Yellow solid; yield: $89.5 \%$; m.p. $141-142{ }^{\circ} \mathrm{C} ;{ }^{1} \mathrm{H}-\mathrm{NMR}$ $\left(600 \mathrm{MHz}, \mathrm{CDCl}_{3}\right) \delta: 8.69(\mathrm{~d}, \mathrm{~J}=7.2 \mathrm{~Hz}, 1 \mathrm{H}), 8.49(\mathrm{~d}$, $J=8.8 \mathrm{~Hz}, 1 \mathrm{H}), 8.36(\mathrm{dd}, J=6.0,3.6 \mathrm{~Hz}, 1 \mathrm{H}), 8.28(\mathrm{dd}$, $\mathrm{J}=6.6,3.6 \mathrm{~Hz}, 1 \mathrm{H}), 8.14(\mathrm{dd}, \mathrm{J}=7.8,1.2 \mathrm{~Hz}, 1 \mathrm{H}), 7.98$ (dd, J=8.4, 7.2 Hz, 1H), 7.94-7.87 (m, 2H), 7.74-7.68 $(\mathrm{m}, 1 \mathrm{H}), 7.51(\mathrm{~d}, \mathrm{~J}=7.8 \mathrm{~Hz}, 1 \mathrm{H}), 7.43(\mathrm{t}, \mathrm{J}=7.8 \mathrm{~Hz}, 1 \mathrm{H})$, $3.86(\mathrm{~s}, 3 \mathrm{H})$. HRMS calcd for $\mathrm{C}_{21} \mathrm{H}_{14} \mathrm{~N}_{2} \mathrm{O}_{4}[\mathrm{M}+\mathrm{H}]^{+}$: 359.1026, found 359.1027.

2-(Ethoxycarbonyl)phenyl phenazine-1-carboxylate (5b) Yellow solid; yield: 92.3\%; m.p. $143-144{ }^{\circ} \mathrm{C}$; ${ }^{1} \mathrm{H}-\mathrm{NMR}$ $\left(600 \mathrm{MHz}, \mathrm{CDCl}_{3}\right)$ 8: 8.74-8.69 (m, $\left.1 \mathrm{H}\right), 8.48(\mathrm{dd}$, $\mathrm{J}=8.4,1.2 \mathrm{~Hz}, 1 \mathrm{H}), 8.36(\mathrm{dd}, \mathrm{J}=6.6,3.6 \mathrm{~Hz}, 1 \mathrm{H}), 8.28$ $(\mathrm{dd}, \mathrm{J}=6.6,3.6 \mathrm{~Hz}, 1 \mathrm{H}), 8.14(\mathrm{dd}, \mathrm{J}=7.8,1.2 \mathrm{~Hz}, 1 \mathrm{H})$, $7.98(\mathrm{dd}, \mathrm{J}=8.4,7.2 \mathrm{~Hz}, 1 \mathrm{H}), 7.93-7.86(\mathrm{~m}, 2 \mathrm{H}), 7.74-$ $7.66(\mathrm{~m}, 1 \mathrm{H}), 7.50(\mathrm{~d}, \mathrm{~J}=7.8 \mathrm{~Hz}, 1 \mathrm{H}), 7.42(\mathrm{t}, \mathrm{J}=7.8 \mathrm{~Hz}$, $1 \mathrm{H}), 4.33(\mathrm{q}, \mathrm{J}=7.2 \mathrm{~Hz}, 2 \mathrm{H}), 1.27(\mathrm{t}, \mathrm{J}=7.2 \mathrm{~Hz}, 3 \mathrm{H})$. HRMS calcd for $\mathrm{C}_{22} \mathrm{H}_{16} \mathrm{~N}_{2} \mathrm{O}_{4}[\mathrm{M}+\mathrm{H}]^{+}: 373.1183$, found 373.1182 .

2-(Propoxycarbonyl)phenyl phenazine-1-carboxylate (5c) Yellow solid; yield: $97.5 \%$; m.p. $102-103{ }^{\circ} \mathrm{C}$; ${ }^{1} \mathrm{H}-\mathrm{NMR}$ $\left(600 \mathrm{MHz}, \mathrm{CDCl}_{3}\right) \delta 8.72(\mathrm{dd}, J=6.6,1.2 \mathrm{~Hz}, 1 \mathrm{H}), 8.48$ $(\mathrm{dd}, J=8.4,1.2 \mathrm{~Hz}, 1 \mathrm{H}), 8.40-8.31(\mathrm{~m}, 1 \mathrm{H}), 8.32-8.21$ $(\mathrm{m}, 1 \mathrm{H}), 8.14(\mathrm{dd}, J=7.8,1.8 \mathrm{~Hz}, 1 \mathrm{H}), 7.98(\mathrm{dd}, J=8.4$, $7.2 \mathrm{~Hz}, 1 \mathrm{H}), 7.94-7.79(\mathrm{~m}, 2 \mathrm{H}), 7.73-7.59(\mathrm{~m}, 1 \mathrm{H}), 7.51$ $(\mathrm{d}, J=7.2 \mathrm{~Hz}, 1 \mathrm{H}), 7.43(\mathrm{dd}, J=11.4,4.2 \mathrm{~Hz}, 1 \mathrm{H}), 4.23$ (t, $J=6.6 \mathrm{~Hz}, 2 \mathrm{H}), 1.74-1.41(\mathrm{~m}, 2 \mathrm{H}), 0.92(\mathrm{t}, J=7.2 \mathrm{~Hz}$, $3 \mathrm{H})$. HRMS calcd for $\mathrm{C}_{23} \mathrm{H}_{18} \mathrm{~N}_{2} \mathrm{O}_{4}[\mathrm{M}+\mathrm{H}]^{+}:$:387.1339, found 387.1338. 
2-(Isopropoxycarbonyl)phenyl phenazine-1-carboxylate (5d) Yellow solid; yield: $90.5 \%$; m.p. $125-126{ }^{\circ} \mathrm{C}$; ${ }^{1} \mathrm{H}-\mathrm{NMR}$ $\left(600 \mathrm{MHz}, \mathrm{CDCl}_{3}\right) \delta 8.73(\mathrm{~d}, J=6.6 \mathrm{~Hz}, 1 \mathrm{H}), 8.48(\mathrm{~d}$, $J=8.4 \mathrm{~Hz}, 1 \mathrm{H}), 8.36(\mathrm{dd}, J=6.6,3.6 \mathrm{~Hz}, 1 \mathrm{H}), 8.27(\mathrm{dd}$, $J=6.6,3.6 \mathrm{~Hz}, 1 \mathrm{H}), 8.12(\mathrm{dd}, J=7.8,1.2 \mathrm{~Hz}, 1 \mathrm{H}), 7.98$ (dd, $J=8.4,7.2 \mathrm{~Hz}, 1 \mathrm{H}), 7.93-7.86(\mathrm{~m}, 2 \mathrm{H}), 7.71-7.66$ $(\mathrm{m}, 1 \mathrm{H}), 7.50(\mathrm{~d}, J=7.8 \mathrm{~Hz}, 1 \mathrm{H}), 7.41(\mathrm{t}, J=7.8 \mathrm{~Hz}, 1 \mathrm{H})$, $5.30-5.30(\mathrm{~m}, 1 \mathrm{H}), 1.27(\mathrm{~d}, J=6.6 \mathrm{~Hz}, 6 \mathrm{H})$. HRMS calcd for $\mathrm{C}_{23} \mathrm{H}_{18} \mathrm{~N}_{2} \mathrm{O}_{4}[\mathrm{M}+\mathrm{H}]^{+}: 387.1339$, found 387.1340.

\section{2-(Butoxycarbonyl)phenyl phenazine-1-carboxylate (5e)}

Yellow solid; yield: 94.1\%; m.p. $89-90{ }^{\circ} \mathrm{C}$; ${ }^{1} \mathrm{H}-\mathrm{NMR}$ $\left(600 \mathrm{MHz}, \mathrm{CDCl}_{3}\right) \delta 8.72(\mathrm{dd}, J=6.9,1.4 \mathrm{~Hz}, 1 \mathrm{H}), 8.48$ (dd, $J=8.7,1.4 \mathrm{~Hz}, 1 \mathrm{H}), 8.39-8.34(\mathrm{~m}, 1 \mathrm{H}), 8.30-8.25(\mathrm{~m}$, $1 \mathrm{H}), 8.13(\mathrm{dd}, J=7.9,1.7 \mathrm{~Hz}, 1 \mathrm{H}), 7.98(\mathrm{dd}, J=8.4,6.6 \mathrm{~Hz}$, $1 \mathrm{H}), 7.93-7.86(\mathrm{~m}, 2 \mathrm{H}), 7.72-7.67(\mathrm{~m}, 1 \mathrm{H}), 7.51(\mathrm{dd}$, $J=7.8,1.2 \mathrm{~Hz}, 1 \mathrm{H}), 7.47-7.37(\mathrm{~m}, 1 \mathrm{H}), 4.27(\mathrm{t}, J=6.7 \mathrm{~Hz}$, $2 \mathrm{H}), 1.72-7.57(\mathrm{~m}, 2 \mathrm{H}), 1.42-1.31(\mathrm{~m}, 2 \mathrm{H}), 0.84(\mathrm{t}$, $J=7.2 \mathrm{~Hz}, 3 \mathrm{H})$. HRMS calcd for $\mathrm{C}_{24} \mathrm{H}_{20} \mathrm{~N}_{2} \mathrm{O}_{4}[\mathrm{M}+\mathrm{H}]^{+}$: 401.1496, found 401.1497.

\section{3-(Methoxycarbonyl)phenyl phenazine-1-carboxylate (5f)}

Yellow solid; yield: $95.0 \%$; m.p. $120-121{ }^{\circ} \mathrm{C}$; ${ }^{1} \mathrm{H}-\mathrm{NMR}$ $\left(600 \mathrm{MHz} \mathrm{CDCl}_{3}\right) \delta 8.48(\mathrm{t}, J=7.2 \mathrm{~Hz}, 2 \mathrm{H}), 8.39-$ $8.34(\mathrm{~m}, 1 \mathrm{H}), 8.30-8.25(\mathrm{~m}, 1 \mathrm{H}), 8.14(\mathrm{~s}, 1 \mathrm{H}), 8.03(\mathrm{~d}$, $J=7.8 \mathrm{~Hz}, 1 \mathrm{H}), 7.98-7.89(\mathrm{~m}, 3 \mathrm{H}), 7.70-7.66(\mathrm{~m}, 1 \mathrm{H})$, $7.59(\mathrm{t}, J=7.8 \mathrm{~Hz}, 1 \mathrm{H}), 3.98(\mathrm{~s}, 3 \mathrm{H})$. HRMS calcd for $\mathrm{C}_{21} \mathrm{H}_{14} \mathrm{~N}_{2} \mathrm{O}_{4}[\mathrm{M}+\mathrm{H}]^{+}:$359.1026, found 359.1027.

\section{3-(Ethoxycarbonyl)phenyl phenazine-1-carboxylate (5g)}

Yellow solid; yield: $96.5 \%$; m.p. $109-110{ }^{\circ} \mathrm{C}$; ${ }^{1} \mathrm{H}-\mathrm{NMR}$ $\left(600 \mathrm{MHz}, \mathrm{CDCl}_{3}\right) \delta 8.55-8.41(\mathrm{~m}, 2 \mathrm{H}), 8.39-8.30(\mathrm{~m}$, $1 \mathrm{H}), 8.31-8.24(\mathrm{~m}, 1 \mathrm{H}), 8.14(\mathrm{~s}, 1 \mathrm{H}), 8.04(\mathrm{~d}, J=7.8 \mathrm{~Hz}$, $1 \mathrm{H}), 7.98-7.85(\mathrm{~m}, 3 \mathrm{H}), 7.67(\mathrm{~d}, J=7.8 \mathrm{~Hz}, 1 \mathrm{H}), 7.59$ (t, $J=7.8 \mathrm{~Hz}, 1 \mathrm{H}), 4.44(\mathrm{q}, J=7.2 \mathrm{~Hz}, 2 \mathrm{H}), 1.44(\mathrm{t}, J=7.2 \mathrm{~Hz}$, $3 \mathrm{H})$. HRMS calcd for $\mathrm{C}_{22} \mathrm{H}_{16} \mathrm{~N}_{2} \mathrm{O}_{4}[\mathrm{M}+\mathrm{H}]^{+}:$373.1183, found 373.1182 .

3-(Propoxycarbonyl)phenyl phenazine-1-carboxylate (5h) Yellow solid; yield: 95.2\%; m.p. 87-88 ${ }^{\circ} \mathrm{C}$; ${ }^{1} \mathrm{H}-\mathrm{NMR}$ $\left(600 \mathrm{MHz}, \mathrm{CDCl}_{3}\right) \quad \delta \quad 8.51-8.43(\mathrm{~m}, 2 \mathrm{H}), \quad 8.38-8.32$ (m, 1H), 8.27 (dd, J=6.0, 4.2 Hz, 1H), 8.13 (s, 1H), 8.04 $(\mathrm{d}, J=7.8 \mathrm{~Hz}, 1 \mathrm{H}), 7.97-7.86(\mathrm{~m}, 3 \mathrm{H}), 7.67(\mathrm{dd}, J=7.8$, $1.2 \mathrm{~Hz}, 1 \mathrm{H}), 7.59(\mathrm{t}, J=7.8 \mathrm{~Hz}, 1 \mathrm{H}), 4.34(\mathrm{t}, J=6.6 \mathrm{~Hz}$, $2 \mathrm{H}), 1.88-1.82(\mathrm{~m}, 1 \mathrm{H}), 1.06(\mathrm{t}, J=7.8 \mathrm{~Hz}, 3 \mathrm{H})$. HRMS calcd for $\mathrm{C}_{23} \mathrm{H}_{18} \mathrm{~N}_{2} \mathrm{O}_{4}[\mathrm{M}+\mathrm{H}]^{+}: 387.1339$, found 387.1340.

\section{3-(Butoxycarbonyl)phenyl phenazine-1-carboxylate (5i)} Yellow solid; yield: 95.5\%; m.p. 97-98 ${ }^{\circ} \mathrm{C}$; ${ }^{1} \mathrm{H}-\mathrm{NMR}$ $\left(600 \mathrm{MHz}, \mathrm{CDCl}_{3}\right) \delta 8.47(\mathrm{~d}, J=7.8 \mathrm{~Hz}, 2 \mathrm{H}), 8.39-8.31$ (m, 1H), 8.29-8.23 (m, 1H), 8.15-8.09 (m, 1H), $8.03(\mathrm{~d}$, $J=7.8 \mathrm{~Hz}, 1 \mathrm{H}), 7.97-7.85(\mathrm{~m}, 3 \mathrm{H}), 7.66(\mathrm{dd}, J=7.8$,
$2.0 \mathrm{~Hz}, 1 \mathrm{H}), 7.58(\mathrm{t}, J=7.8 \mathrm{~Hz}, 1 \mathrm{H}), 5.37-5.25(\mathrm{~m}, 1 \mathrm{H})$, $1.41(\mathrm{~d}, J=6.6 \mathrm{~Hz}, 6 \mathrm{H})$. HRMS calcd for $\mathrm{C}_{23} \mathrm{H}_{18} \mathrm{~N}_{2} \mathrm{O}_{4}$ $[\mathrm{M}+\mathrm{H}]^{+}:$387.1339, found 387.1340.

\section{3-(Butoxycarbonyl)phenyl phenazine-1-carboxylate (5j)} Yellow solid; yield: 95.2\%; m.p. $87-88{ }^{\circ} \mathrm{C}$; ${ }^{1} \mathrm{H}-\mathrm{NMR}$ $\left(600 \mathrm{MHz}, \mathrm{CDCl}_{3}\right) \delta 8.48(\mathrm{dd}, J=7.8,3.6 \mathrm{~Hz}, 2 \mathrm{H})$, 8.39-8.33 (m, 1H), 8.31-8.25 (m, 1H), 8.12 (s, 1H), 8.04 $(\mathrm{d}, J=7.8 \mathrm{~Hz}, 1 \mathrm{H}), 7.98-7.90(\mathrm{~m}, 3 \mathrm{H}), 7.67(\mathrm{dd}, J=7.8$, $2.4 \mathrm{~Hz}, 1 \mathrm{H}), 7.59(\mathrm{t}, J=7.8 \mathrm{~Hz}, 1 \mathrm{H}), 4.39(\mathrm{t}, J=6.6 \mathrm{~Hz}$, $2 \mathrm{H}), 1.83-1.77(\mathrm{~m}, 2 \mathrm{H}), 1.56-1.48(\mathrm{~m}, 2 \mathrm{H}), 1.01(\mathrm{t}$, $J=7.2 \mathrm{~Hz}, 3 \mathrm{H})$. HRMS calcd for $\mathrm{C}_{24} \mathrm{H}_{20} \mathrm{~N}_{2} \mathrm{O}_{4}[\mathrm{M}+\mathrm{H}]^{+}$: 401.1496, found 401.1495.

\section{4-(Methoxycarbonyl)phenyl phenazine-1-carboxylate (5k)} Yellow solid; yield: $95.0 \%$; m.p. $164-165{ }^{\circ} \mathrm{C}$; ${ }^{1} \mathrm{H}-\mathrm{NMR}$ $\left(600 \mathrm{MHz}, \mathrm{CDCl}_{3}\right) \delta 8.54-8.43(\mathrm{~m}, 2 \mathrm{H}), 8.37-8.33(\mathrm{~m}$, $1 \mathrm{H}), 8.31-8.26(\mathrm{~m}, 1 \mathrm{H}), 8.24-8.18(\mathrm{~m}, 2 \mathrm{H}), 7.97-7.90$ (m, 3H), 7.57-7.51 (m, 2H), 3.97 (s, 3H). HRMS calcd for $\mathrm{C}_{21} \mathrm{H}_{14} \mathrm{~N}_{2} \mathrm{O}_{4}[\mathrm{M}+\mathrm{H}]^{+}:$359.1026, found 359.1025.

4-(Ethoxycarbonyl)phenyl phenazine-1-carboxylate (5I) Yellow solid; yield: 98.1\%; m.p. $123-125{ }^{\circ} \mathrm{C}$; ${ }^{1} \mathrm{H}-\mathrm{NMR}$ $\left(600 \mathrm{MHz}, \mathrm{CDCl}_{3}\right) \delta 8.51-8.46(\mathrm{~m}, 2 \mathrm{H}), 8.38-8.32(\mathrm{~m}$, $1 \mathrm{H}), 8.31-8.26(\mathrm{~m}, 1 \mathrm{H}), 8.21(\mathrm{t}, J=5.4 \mathrm{~Hz}, 2 \mathrm{H}), 7.98-7.89$ $(\mathrm{m}, 3 \mathrm{H}), 7.53(\mathrm{t}, J=5.4 \mathrm{~Hz}, 2 \mathrm{H}), 4.43(\mathrm{q}, J=7.2 \mathrm{~Hz}, 2 \mathrm{H})$, $1.44(\mathrm{t}, J=7.2 \mathrm{~Hz}, 3 \mathrm{H})$. HRMS calcd for $\mathrm{C}_{22} \mathrm{H}_{16} \mathrm{~N}_{2} \mathrm{O}_{4}$ $[\mathrm{M}+\mathrm{H}]^{+}:$373.1183, found 373.1182.

4-(Propoxycarbonyl)phenyl phenazine-1-carboxylate (5m) Yellow solid; yield: $98.1 \%$; m.p. $95{ }^{\circ} \mathrm{C}$; ${ }^{1} \mathrm{H}-\mathrm{NMR}(600 \mathrm{MHz}$, $\left.\mathrm{CDCl}_{3}\right) \delta 8.61-8.40(\mathrm{~m}, 2 \mathrm{H}), 8.41-8.30(\mathrm{~m}, 1 \mathrm{H}), 8.31-$ $8.27(\mathrm{~m}, 1 \mathrm{H}), 8.27-8.15(\mathrm{~m}, 2 \mathrm{H}), 8.02-7.82(\mathrm{~m}, 3 \mathrm{H})$, 7.64-7.46 (m, 2H), $4.33(\mathrm{t}, J=6.6 \mathrm{~Hz}, 2 \mathrm{H}), 1.89-1.79(\mathrm{~m}$, $2 \mathrm{H}), 1.07(\mathrm{t}, J=7.2 \mathrm{~Hz}, 3 \mathrm{H})$. HRMS calcd for $\mathrm{C}_{23} \mathrm{H}_{18} \mathrm{~N}_{2} \mathrm{O}_{4}$ $[\mathrm{M}+\mathrm{H}]^{+}:$387.1339, found 387.1340.

4-(Butoxycarbonyl)phenyl phenazine-1-carboxylate (5n) Yellow solid; yield: 97.5\%; m.p. $119-120{ }^{\circ} \mathrm{C}$; ${ }^{1} \mathrm{H}-\mathrm{NMR}$ $\left(600 \mathrm{MHz}, \mathrm{CDCl}_{3}\right) \delta 8.52-8.44(\mathrm{~m}, 2 \mathrm{H}), 8.36-8.31(\mathrm{~m}$, $1 \mathrm{H}), 8.30-8.25(\mathrm{~m}, 1 \mathrm{H}), 8.23-8.18(\mathrm{~m}, 2 \mathrm{H}), 7.96-7.89$ $(\mathrm{m}, 3 \mathrm{H}), 7.55-7.51(\mathrm{~m}, 2 \mathrm{H}), 5.33-5.28(\mathrm{~m}, 1 \mathrm{H}), 1.41(\mathrm{~d}$, $J=6.6 \mathrm{~Hz}, 6 \mathrm{H})$. HRMS calcd for $\mathrm{C}_{23} \mathrm{H}_{18} \mathrm{~N}_{2} \mathrm{O}_{4}[\mathrm{M}+\mathrm{H}]^{+}$: 387.1339 , found 387.1340 .

\section{4-(Butoxycarbonyl)phenyl phenazine-1-carboxylate (5o)}

Yellow solid; yield: 99.0\%; m.p. $89-90{ }^{\circ} \mathrm{C}$; ${ }^{1} \mathrm{H}-\mathrm{NMR}$ $\left(600 \mathrm{MHz}, \mathrm{CDCl}_{3}\right) \delta 8.53-8.39(\mathrm{~m}, 2 \mathrm{H}), 8.36-8.31(\mathrm{~m}$, $1 \mathrm{H}), 8.29-8.25(\mathrm{~m}, 1 \mathrm{H}), 8.24-8.19(\mathrm{~m}, 2 \mathrm{H}), 7.96-7.87$ $(\mathrm{m}, 3 \mathrm{H}), 7.56-7.51(\mathrm{~m}, 2 \mathrm{H}), 4.38(\mathrm{t}, J=6.6 \mathrm{~Hz}, 2 \mathrm{H}), 1.88-$ $1.76(\mathrm{~m}, 2 \mathrm{H}), 1.57-1.48(\mathrm{~m}, 2 \mathrm{H}), 1.02(\mathrm{t}, J=7.2 \mathrm{~Hz}, 3 \mathrm{H})$. HRMS calcd for $\mathrm{C}_{24} \mathrm{H}_{20} \mathrm{~N}_{2} \mathrm{O}_{4}[\mathrm{M}+\mathrm{H}]^{+}: 401.1496$, found 401.1497. 
4-(Octyloxycarbonyl)phenyl phenazine-1-carboxylate (5p) Yellow solid; yield: 97.1\%; m.p. $57-59{ }^{\circ} \mathrm{C}$; ${ }^{1} \mathrm{H}-\mathrm{NMR}$ $\left(600 \mathrm{MHz}, \mathrm{CDCl}_{3}\right) \delta 8.60-8.40(\mathrm{~m}, 2 \mathrm{H}), 8.43-8.31(\mathrm{~m}$, $1 \mathrm{H}), 8.31-8.24(\mathrm{~m}, 1 \mathrm{H}), 8.25-8.18(\mathrm{~m}, 2 \mathrm{H}), 8.02-7.85(\mathrm{~m}$, $3 \mathrm{H}), 7.63-7.46(\mathrm{~m}, 2 \mathrm{H}), 4.36(\mathrm{t}, J=6.6 \mathrm{~Hz}, 2 \mathrm{H}), 1.88-1.76$ (m, 2H), 1.53-1.43 (m, 2H), 1.42-1.26 (m, 8H), $0.91(\mathrm{t}$, $J=6.6 \mathrm{~Hz}, 3 \mathrm{H})$. HRMS calcd for $\mathrm{C}_{28} \mathrm{H}_{28} \mathrm{~N}_{2} \mathrm{O}_{4}[\mathrm{M}+\mathrm{H}]^{+}$: 457.2122, found 457.2123.

\section{Biological assays}

Compounds were screened for their in vitro fungicidal activity against Rhizoctonia solani, Fusaium graminearum, Altemaria solani, Fusarium oxysporum, Sclerotinia sclerotiorum and Pyricularia oryzae with the mycelium growth rate test.

The method for testing the primary biological activity was performed aseptically with pure cultures. Synthesized compounds were dissolved in $100 \%$ acetone, and the solutions were diluted with aqueous $1 \%$ Tween 80 and were then added to sterile potato dextrose agar (PDA). The target final concentration of each compound was $50 \mu \mathrm{g} / \mathrm{mL}$. The control blank assay was performed with $1 \mathrm{~mL}$ of sterile water. Mycelial plugs $6 \mathrm{~mm}$ in diameter were obtained with a cork borer and placed on the amended PDA. The culture plates were incubated at $28{ }^{\circ} \mathrm{C}$. The diameter of the mycelia was measured after $72 \mathrm{~h}$. Acetone in sterile aqueous 1\% Tween 80 served as the negative control, whereas phenazine-1-carboxylic acid served as positive controls. Each sample was screened with three replicates, and each colony diameter of the three replicates was measured four times. All statistical analysis was performed using EXCEL 2010 software. The log dose-response curves allowed determination of the $\mathrm{EC}_{50}$ for the bioassay using probit analysis. The $95 \%$ confidence limits for the range of $\mathrm{EC}_{50}$ values were determined by the least-square regression analysis of the relative growth rate (\% control) against the logarithm of the compound concentration. The relative inhibition rate of the circle mycelium compared to blank assay was calculated via the following equation:

$$
\begin{aligned}
& \text { Relative inhibition rate }(\%) \\
& \quad=[(\mathrm{CK}-\mathrm{PT}) /(\mathrm{CK}-6 \mathrm{~mm})] \times 100 \%
\end{aligned}
$$

where $\mathrm{CK}$ is the extended diameter of the circle mycelium during the blank assay; and PT is the extended diameter of the circle mycelium during testing.

\section{Plant materials and fungal growth condition}

Seeds of rice (Feng liang you xiang No. 1), with high rates of germination, were grown in plastic pots of $20 \mathrm{~cm}$ diameter and kept in a greenhouse under a temperature of $26-28{ }^{\circ} \mathrm{C}$, with 10 plant per pot. After 4 weeks the four-leaf stage plants were used in the experiments. Rhizoctonia solani was cultured for 4 days at $28{ }^{\circ} \mathrm{C}$ on potato dextrose agar (PDA), under aseptic conditions. Spore concentration was adjusted with sterile distilled water to $10^{5}$ spores $/ \mathrm{mL}$.

\section{Chemical treatment of plants}

Chemical treatments of plants were carried out as described by Makandar and others [34, 35]. Briefly, a stock solution of $10 \mathrm{mmol} / \mathrm{L}$ for testing conjugate 5c (highest fungicidal activity against Rhizoctonia solani) was prepared in water and diluted to a final concentration of $200 \mu \mathrm{mol} / \mathrm{L}$. Rice plants at the four-leaf of the similar size were sprayed with a concentration of $200 \mu \mathrm{mol} / \mathrm{L}$ of test conjugate 5c, PCA and of salicylic acid (SA). A blank water control was also applied under the same conditions. There were four treatments as follows: (1) PCA, (2) SA, (3) conjugate 5c, and water-treated control. Each treatment consisted of three pots each containing 10 rice seedlings, and were arranged in a completely randomized design and replicated four times. In all treatments, spraying was done $24 \mathrm{~h}$ prior to inoculation.

\section{Fungal inoculation and disease rating}

Plants were treated with chemicals and $24 \mathrm{~h}$ later, point inoculations of rice leaf sheaths were done with needle injection of $10 \mu \mathrm{L}$ of the $10^{5}$ spores $/ \mathrm{mL}$ suspension at the four-leaf stage of seedlings of rice. For each replication of each treatment, 30 leaf sheaths were inoculated. The inoculated plants were covered with black plastic bags and kept in a growth room maintained at $90 \%$ relative humidity near $90 \%$ at $26-28{ }^{\circ} \mathrm{C}$ for $24 \mathrm{~h}$. Plants were evaluated for rice sheath blight disease as percent leaf sheath infected with Rhizoctonia solani at 14 days after inoculation. All statistical analyses were performed using EXCEL 2010 software. The disease reduction was calculated as follows:

$$
\text { Disease reduction }(\%)=[(\mathrm{CK}-\mathrm{PT}) / \mathrm{CK}] \times 100 \%
$$

where $\mathrm{CK}$ is the percent disease in inoculated plants treated with water while $\mathrm{PT}$ is the disease rating for inducer treatments.

\section{Conclusions}

In summary, we prepared 16 novel hydroxybenzoic acid ester conjugates of phenazine-1-carboxylic acid and investigated their biological activity. Most of the synthetic conjugates displayed some level of fungicidal activity in vitro against five phytopathogenic fungi. In particular, nine conjugates $\mathbf{5 b}, \mathbf{5 c}, \mathbf{5 d}, \mathbf{5 e}, \mathbf{5 h}, \mathbf{5 i}, \mathbf{5 m}$, 5n and $5 \mathbf{o}\left(\mathrm{EC}_{50}\right.$ values were between $3.2 \mu \mathrm{g} / \mathrm{mL}$ and $14.1 \mu \mathrm{g} / \mathrm{mL}$ ) were more active than PCA $\left(\mathrm{EC}_{50}\right.$ value was $18.6 \mu \mathrm{g} / \mathrm{mL}$ ) against Rhizoctonia solani, and conjugate $\mathbf{5 c}$ 
had the highest fungicidal activity, 6.5-fold greater than PCA. The results of the bioassay indicated that the fungicidal activity of conjugates is associated with their LogP, and the optimal $\log \mathrm{P}$ values of the more potent fungicidal activity within these conjugates ranged from 4.42 to 5.08. The test of systemic acquired resistance against rice sheath blight disease in rice seedlings revealed that PCA-SA ester conjugate $\mathbf{5 c}$ retains the resistance induction activity of SA to rice sheath blight, and has higher activity than SA. Meanwhile, the mechanism of systemic acquired resistance against rice sheath blight in rice seedlings by PCA-SA ester conjugate $\mathbf{5 c}$ will be the focus of our next study.

\section{Additional file}

Additional file 1. Spectrum data of PCA derivatives. Which includes the copies of $1 \mathrm{H}$ NMR and HRMS of selected compounds.

\section{Authors' contributions}

The current study is an outcome of constructive discussion with $J L$ and XZ. XZ synthesized the compounds and carried out most of the bioassay experiments. LY, MZ and ZY did part of the bioassay experiments. XZ took part in the compound structural elucidation and bioassay experiments. ZX and QW carried out some structure elucidation experiments. JL was the principle investigator of the project and provided the research funding. XD is the cocorresponding author for this work. All authors read and approved the final manuscript.

\section{Author details}

${ }^{1}$ Hubei Collaborative Innovation Centre for Grain Industry, Yangtze University, Jingmi Road 88, Jingzhou 434025, China. ${ }^{2}$ School of Agriculture, Yangtze University, Jingmi Road 88, Jingzhou 434025, China. ${ }^{3}$ Engineering Research Center of Ecology and Agricultural Use of Wetland, Ministry of Education, Yangtze University, Jingmi Road 88, Jingzhou 434025, China.

\section{Competing interests}

The authors declare that they have no competing interests.

\section{Availability of data and materials}

We have presented all our main data in the form of tables and figures. Meanwhile, all the copies of ${ }^{1} \mathrm{H}$ NMR and HRMS for the title compounds were presented in the Additional file.

\section{Funding and acknowledgements}

The authors gratefully acknowledge Grants from the National Natural Science Foundation of China (No. 31672069) and Natural Science Foundation of Hubei Province (No. 2014CFA105).

\section{Publisher's Note}

Springer Nature remains neutral with regard to jurisdictional claims in published maps and institutional affiliations.

Received: 3 August 2018 Accepted: 19 October 2018 Published online: 01 November 2018

\section{References}

1. Laursen JB, Nielsen J (2004) Phenazine natural products: biosynthesis, synthetic analogues, and biological activity. Chem Rev 104:1663
2. Guttenberger N, Blankenfeldt W, Breinbauer R (2017) Recent developments in the isolation, biological function, biosynthesis, and synthesis of phenazine natural products. Bioorg Med Chem 25:6149-6166

3. Messenger AJM, Turner JM (1983) Phenazine-1, 6-dicarboxylate and its dimethyl ester as precursors of other phenazines in bacteria. FEMS Microbiol Lett 18:65-68

4. Pusecker K, Laatsch H, Helmke E, Weyland H (1997) Dihydrophencomycin methyl ester, a new phenazine derivative from a marine Streptomycetet. J Antibiot 50:479-483

5. Puopolo G, Masi M, Raio A, Andolfi A, Zoina A, Cimmino A, Evidente A (2013) Insights on the susceptibility of plant pathogenic fungi to phenazine-1-carboxylic acid and its chemical derivatives. Nat Prod Res 27:956-966

6. Bigge CF, Elslager EF, French JC, Graham BD, Hokanson GC, Mamber SW Smitka TA, Tunac JB, Wilton JH (1987) Antimicrobial and antitumor phenazine carboxaldehydes and derivatives. US 4657909

7. Palchykovska LG, Vasylchenko OV, Platonov MO, Kostina VG, Babkina MM, Tarasov OA, Starosyla DB, Samijlenko SP, Rybalko SL, Deriabin OM, Hovorun DM (2012) Evaluation of antibacterial and antiviral activity of $\mathrm{N}$-arylamides of 9-methyl and 9-methoxyphenazine-1-carboxylic acids inhibitors of the phage T7 model transctiption. Biopolym Cell 28:477-485

8. Udumula V, Endres JL, Harper CN, Jaramillo L, Zhong HA, Bayles KW, Conda-Sheridan M (2016) Simple synthesis of endophenazine $G$ and other phenazines and their evaluation as anti-methicillin-resistant Staphylococcus aureus agents. Eur J Med Chem 125:710-721

9. Rewcastle GW, Denny WA, Baguley BC (1987) Potential antitumor agents. 51. Synthesis and antitumor activity of substituted phenazine-1-carboxamides. J Med Chem 30:843-851

10. Mekapati SB, Denny WA, Kurupa A, Hanscha C (2001) QSAR of anticancer compounds. bis(11-oxo-11H-indeno[1,2-b]quinoline-6-carboxamides), bis(phenazine-1-carboxamides) and bis(naphthalimides). Bioorg Med Chem 9:2757-2762

11. Spicer JA, Gamage SA, Rewcastle GW, Finlay GJ, Bridewell DJA, Baguley BC, Denny WA (2000) Bis(phenazine-1-carboxamides): structure-activity relationships for a new class of dual topoisomerase I/II-directed anticancer drugs. J Med Chem 43:1350-1358

12. Gamage SA, Rewcastle GW, Baguley BC, Charltonb PA, Denny WA (2006) Phenazine-1-carboxamides: structure-cytotoxicity relationships for 9-substituents and changes in the $\mathrm{H}$-bonding pattern of the cationic side chain. Bioorg Med Chem 14:1160-1168

13. Gupta A, Jaiswal A, Prachnad S (2014) Quantitative structure activity relationship analysis of $\mathrm{N}$-substituted phenazine-1-carboxamides analogs as anti-mycobacterial agents. Int J Pharm Life Sci 5:3230-3240

14. Logua AD, Palchykovska LH, Kostina VH, Sanna A, Meleddu R, Chisu L, Alexeeva IV, Shved AD (2009) Novel N-aryl- and N-heteryl-phenazine1-carboxamides as potential agents for the treatment of infections sustained by drug-resistant and MDR Mycobacterium tuberculosis. Int J Antimicrob Agents 33:223-229

15. Commare RR, Nandakumar R, Kandan A, Suresh S, Bharathi M, Raguchander T, Samiyappan R (2002) Pseudomonas fluorescens based bio-formulation for the management of sheath blight disease and leaffolder insect in rice. Crop Prot 21:671-677

16. Zhou Q, Su J, Jiang H, Huang X, Xu Y (2010) Optimization of phenazine1-carboxylic acid production by a gacA/qscR-inactivated Pseudomonas sp. M18GQ harboring pME6032Phz using response surface methodology. Appl Microbiol Biot 86:1761-1773

17. Ye L, Zhang H, Xu H, Zou Q, Cheng C, Dong D, Xu Y, Li R (2010) Phenazine1-carboxylic acid derivatives: design, synthesis and biological evaluation against Rhizoctonia solani Kuhn. Bioorg Med Chem Lett 20:7369-7371

18. Su JJ, Zhou Q, Zhang HY, Li YQ, Huang XQ, Xu YQ (2010) Medium optimization for phenazine-1-carboxylic acid production by a gacA qscR double mutant of Pseudomonas sp. M18 using response surface methodology. Bioresour Technol 101:4089-4095

19. Chin-A-Woeng TFC, Bloemberg GV, Lugtenberg BJJ (2003) Phenazines and their role in biocontrol by Pseudomonas bacteria. New Phytol 157:503-523

20. Li B, Lu L, Sun Q, Zhu QD, Li ZN, Wang G (2016) CN105418518A

21. Wang MZ, Xu H, Yu SJ, Feng Q, Wang SH, Li ZM (2010) Synthesis and fungicidal activity of novel aminophenazine-1-carboxylate derivatives. J Agric Food Chem 58:3651-3660 
22. Gaffney T, Friedrich L, Vernooij B, Negrotto D, Nye G, Uknes S, Ward E, Kessmann H, Ryals J (1993) Requirement of salicylic acid for the induction of systemic acquired resistance. Science 261:754-756

23. Poóra P, Takács Z, Bela K, Czékus Z, Szalai G, Tari I (2017) Prolonged dark period modulates the oxidative burst and enzymatic antioxidant systems in the leaves of salicylic acid-treated tomato. J Plant Physiol 213:216-226

24. Bernsdorff F, Döring AC, Gruner K, Schuck S, Bräutigam A, Zeier J (2016) Pipecolic acid orchestrates plant systemic acquired resistance and defense priming via salicylic acid-dependent and -independent pathways. Plant Cell 28:102-129

25. Silverman FP, Petracek PD, Heiman DF, Fledderman CM, Warrior P (2005) Salicylate activity. 3. Structure relationship to systemic acquired resistance. J Agric Food Chem 53:9775-9780

26. Safari S, Soleimani MJ, Mohajer A, Fazlikhani L (2013) Possible structureactivity profile of salicylate derivatives: their relationship on induction of systemic acquired resistance. J Agric Technol 9:1215-1225

27. Cueva C, Moreno-Arribas MV, Martín-Álvarez PJ, Bills G, Vicente MF, Basilio A, Rivas CL, Requena T, Rodríguez JM, Bartolome B (2010) Antimicrobial activity of phenolic acids against commensal, probiotic and pathogenic bacteria. Res Microbiol 161:372-382

28. Chong KP, Rossall S, Atong M (2009) In vitro antimicrobial activity and fungitoxicity of syringic acid, caffeic acid and 4-hydroxybenzoic acid against Ganoderma Boninense. J Agric Sci 1:15-20
29. Kosová M, Hrádková I, Mátlová V, Kadlec D, Šmidrkal J, Filip V (2015) Antimicrobial effect of 4-hydroxybenzoic acid ester with glycerol. J Clin Pharm Ther 40:436-440

30. Özçelik B, Kartal M, Orhan I (2011) Cytotoxicity, antiviral and antimicrobial activities of alkaloids, flavonoids, and phenolic acids. Pharm Biol 49:396-402

31. Flausino OA Jr, Dufau L, Regasini LO, Petrônio MS, Silva DH, Rose T, Bolzani VS, Reboud-Ravaux M (2012) Alkyl hydroxybenzoic acid derivatives that inhibit HIV-1 protease dimerization. Curr Med Chem 19:4534-4540

32. Manuja R, Sachdeva S, Jain A, Chaudhary J (2013) A comprehensive review on biological activities of P-hydroxy benzoic acid and its derivatives. Int J Pharm Sci Rev Res 22:109-115

33. Price-Whelan A, Dietrich LEP, Newman DK (2007) Pyocyanin alters redox homeostasis and carbon flux through central metabolic pathways in Pseudomonas aeruginosa PA14. J Bacteriol 189:6372-6381

34. Makandar R, Nalam VJ, Lee H, Trick HN, Dong Y, Shah J (2012) Salicylic acid regulates basal resistance to Fusarium head blight in wheat. Mol Plant Microbe Interact 25:431-439

35. Sorahinobar M, Niknam V, Ebrahimzadeh $H$, Soltanloo H, Behmanesh M, Enferadi ST (2016) Central role of salicylic acid in resistance of wheat against Fusarium graminearum. J Plant Growth Regul 35:477-491
Ready to submit your research? Choose BMC and benefit from:

- fast, convenient online submission

- thorough peer review by experienced researchers in your field

- rapid publication on acceptance

- support for research data, including large and complex data types

- gold Open Access which fosters wider collaboration and increased citations

- maximum visibility for your research: over 100M website views per year

At BMC, research is always in progress.

Learn more biomedcentral.com/submissions 